IdeAs

Idées d'Amériques

15 | 2020

Eau et gestion de l'eau dans les Amériques

\title{
Sécurité de l'eau et changement climatique au Brésil : entretien croisé avec Jerson Kelman et João Paulo Capobianco
}

Livia Kalil et Guilherme B. Checco

Traducteur : Livia Kalil

\section{OpenEdition}

\section{Journals}

Édition électronique

URL : http://journals.openedition.org/ideas/7359

DOI : 10.4000/ideas.7359

ISSN : 1950-5701

\section{Éditeur}

Institut des Amériques

\section{Référence électronique}

Livia Kalil et Guilherme B. Checco, « Sécurité de l'eau et changement climatique au Brésil : entretien croisé avec Jerson Kelman et João Paulo Capobianco », IdeAs [En ligne], 15 | 2020, mis en ligne le 01 mars 2020, consulté le 25 mars 2020. URL : http://journals.openedition.org/ideas/7359 ; DOI : https:// doi.org/10.4000/ideas.7359

Ce document a été généré automatiquement le 25 mars 2020.

\section{cc)}

IdeAs - Idées d'Amériques est mis à disposition selon les termes de la licence Creative Commons Attribution - Pas d'Utilisation Commerciale - Pas de Modification 4.0 International. 
Sécurité de l'eau et changement climatique au Brésil : entretien croisé avec Jerson Kelman et João Paulo Capobianco

Livia Kalil et Guilherme B. Checco

Traduction : Livia Kalil

Jerson Kelman

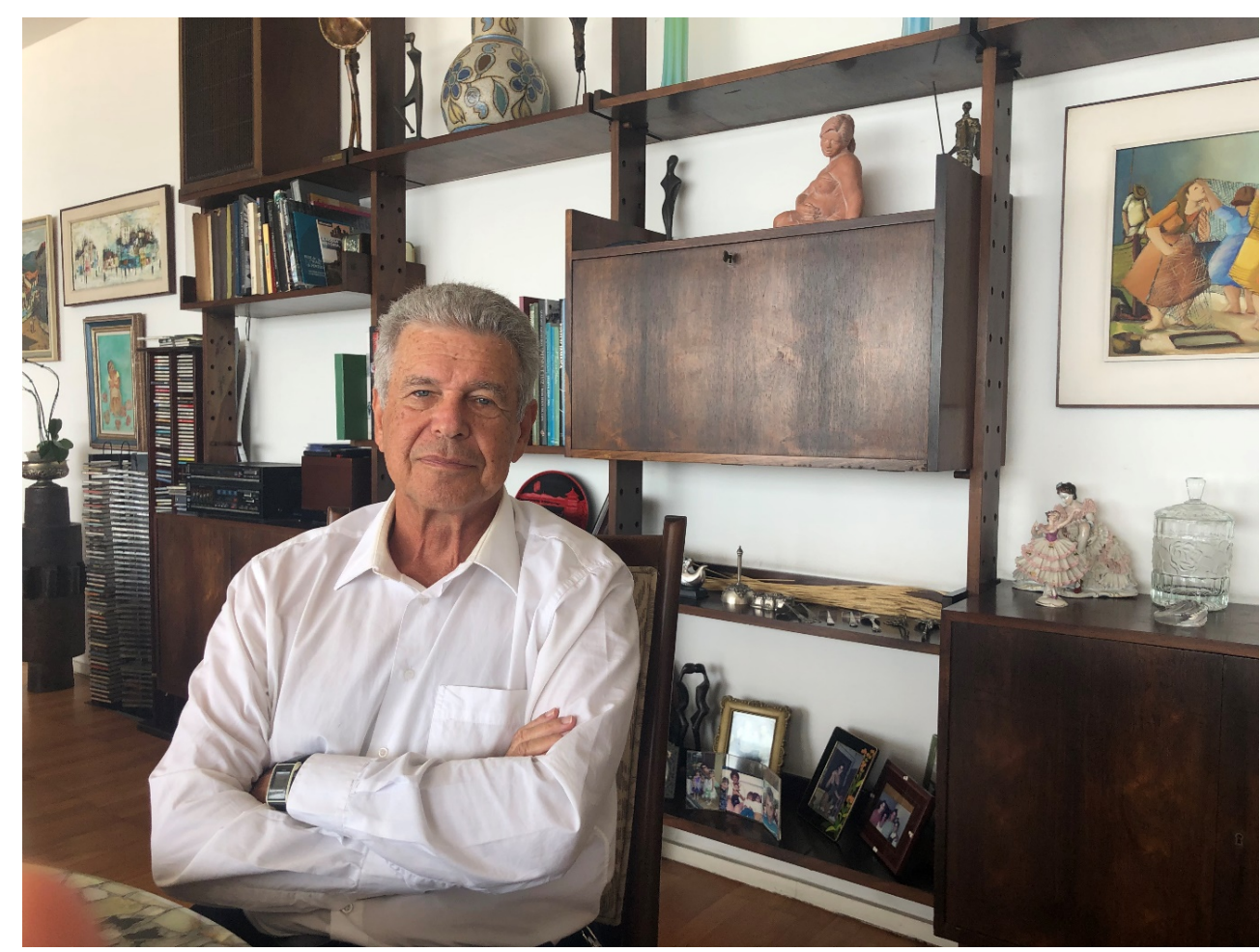

(c) Livia Kalil 
João Paulo Capobianco

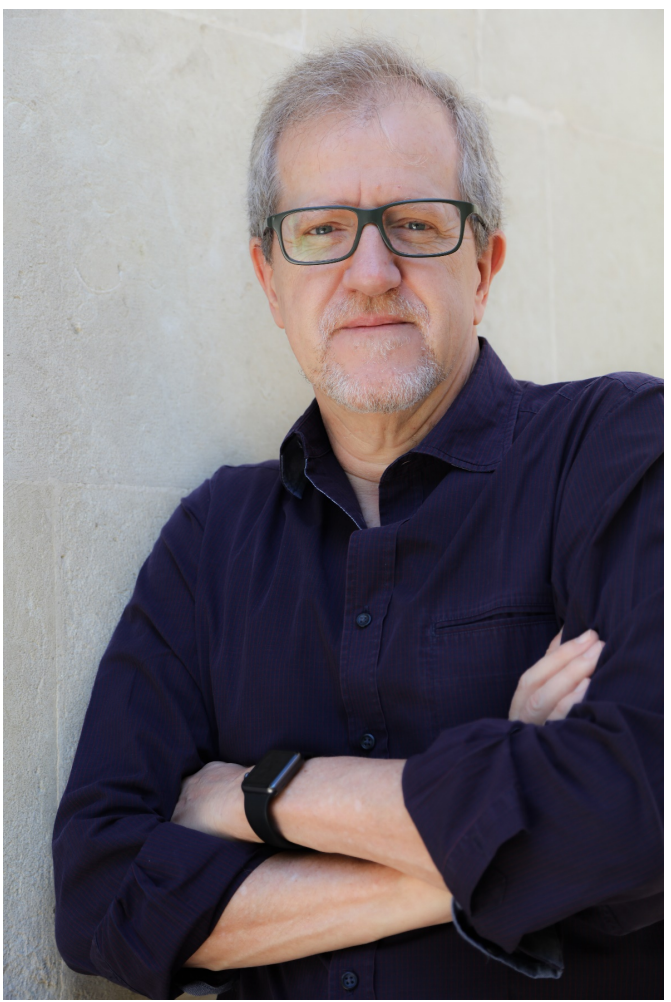

(C) Luciana Capobianco

1 Les scientifiques sont catégoriques en affirmant que l'un des principaux impacts du changement climatique est sur le cycle hydrologique (IPCC, 2014 et 2018). Le Brésil est un pays qui joue un rôle stratégique dans cet enjeu. En plus d'être parmi les plus grands émetteurs de gaz à effet de serre au monde (plus précisément le $7^{\mathrm{e}}$ ), ayant la plus grande et plus biodiverse forêt tropicale, il est aussi le pays détenteur de la plus grande réserve d'eau douce au monde, arrivant à $12 \%$ du total. Or, les impacts des changements climatiques et des évènements extrêmes hydrologiques font déjà partie de la réalité brésilienne (Marengo, 2008). Sur la seule année 2018, un million de Brésiliens ont été touchés par des inondations, tandis que près de 43 millions ont été touchés par des sécheresses (ANA, 2019). Comprendre un tel défi est une tâche qui exige un regard stratégique et multidisciplinaire.

2 Ainsi, nous avons décidé d'interviewer deux spécialistes dans ce domaine, qui ont consacré leurs travaux au Brésil, en particulier à cette problématique. Jerson Kelman et João Paulo Capobianco ont déjà travaillé ensemble au sein du ministère de l'Environnement. Ils ont tout de même gardé leurs idées, leurs particularités ainsi que leurs champs politiques spécifiques. Dans cet entretien croisé, les spécialistes dressent un diagnostic approfondi de la conjoncture et proposent des pistes pour la gestion de l'eau au Brésil face au changement climatique avec une intéressante complémentarité. Jerson Kelman est ingénieur de formation, professeur à l'Universidade Federal do Rio de Janeiro (UFRJ) et ex-Directeur président de l'Agence nationale de l'eau (ANA). João Paulo Capobianco est biologiste de formation, environnementaliste, responsable de la fondation de plusieurs organisations sociales comme la Fondation SOS Mata Atlântica 
et l'Instituto Socioambiental (ISA) et ex-secrétaire exécutif du ministère de l'Environnement.

LiVIA KAlil et GUiLherme B. CHecco (L.K., G.B.) : Comment se passe la gouvernance de l'eau au Brésil ?

Jerson Kelman (J.K.) : Lorsqu'on parle de l'eau, pour commencer, il faut faire une distinction : c'est une chose de gérer l'eau douce dans son état "brut ", l'eau des rivières, des réservoirs; cela en est une autre de gérer le service d'assainissement. Parfois, ces deux choses sont mélangées.

En ce qui concerne la première, la gestion de l'eau en tant que ressource naturelle, au Brésil, selon la Constitution fédérale (1988), les eaux qui font frontière entre des États ou pays sont du ressort de l'Union. La Constitution est un peu vague sur ce que seraient les autres eaux. Ce doute a été comblé par l'extension d'une compréhension existante depuis les Constitutions précédentes, qui soutenaient que les rivières qui se déversent dans le même État seraient de la responsabilité de cet État.

À mon avis, le Brésil n'a malheureusement pas adopté un système unique d'attribution de la responsabilité gouvernementale en matière des eaux. Comme c'est le cas de la France, par exemple, un État qui n'est pas une fédération. Ainsi, en France, il est certainement plus facile de gérer l'eau qu'au Brésil. Le Brésil souffre d'une complication à cet égard, qui touche les pays fédérés (comme c'est le cas des États-Unis, de l'Australie). La difficulté est que lorsque vous avez la responsabilité de la gestion de la ressource naturelle divisée dans un même bassin versant entre différentes entités, cela crée une difficulté technique et administrative. Nous savons qu'une goutte d'eau, dans un bassin versant, ne sait pas où elle est tombée, et qu'elle va, selon l'interprétation de la Constitution, être un moment sous l'administration de l'État A, puis, à un autre moment sous le contrôle de l'État B, et puis sous la responsabilité du gouvernement fédéral. De nombreuses autorités sont responsables de la même ressource.

L'unité de gestion devrait être celle du bassin versant, parce que l'équilibre entre l'offre et la demande, en termes quantitatifs et qualitatifs, exige d'examiner toutes les utilisations du bassin, décisions prises dans un affluent, qu'affectera la disponibilité de l'eau en aval. Donc cette double domination [entre l'Union et les différents États] est très difficile à mettre en œuvre.

João Paulo Capobianco (JPC) : D'un point de vue théorique, la conception de la gouvernance de l'eau au Brésil, et spécialement la politique d'assainissement de base, est assez avancée et, en thèse, elle devrait, si elle était bien appliquée, être assez efficace. Elle présuppose une exigence de planification, avec des processus participatifs, en articulant les principaux acteurs impliqués dans la question de l'usage de l'eau, avec un rôle important pour les municipalités qui sont celles qui ont la responsabilité de la gestion de l'eau. C'est-à-dire qu'il s'agit d'un instrument qui contiendrait les éléments fondamentaux pour une gestion négociée, démocratique et donc avec des chances d'engendrer de bons résultats.

En d'autres termes, les instruments sont assez avancés, mais ce que nous pouvons constater, c'est qu'ils ne produisent pas les résultats souhaités [au Brésil la moitié de la population, environ 100 millions de personnes, n'a pas d'accès à la collecte et au traitement des eaux usées]. 
L.K., G.B.: Quels sont les principaux défis de la gestion des ressources en eau du pays?

J.K. : Le premier défi est un événement commun à tous les pays développés qui aujourd'hui ont déjà résolu ce problème : la pollution. C'est-à-dire le défi de la mise en place d'une infrastructure de collecte et de traitement des eaux usées. La France a été confrontée à ce problème dans les années 1960 et l'a géré en créant la redevance de pollution et les agences de bassin. Cela a été fait en imposant les citoyens français, qui ont commencé à payer cette taxe sur leur facture d'eau. Dans mon lointain souvenir de ce qui s'est passé en France, cela a fait que les agences de bassin ont développé un grand pouvoir économique parce qu'elles ont pu lever des fonds importants. Donc, c'est le citoyen français lui-même qui a permis à la France, avec la redevance, d'en finir avec la pollution des fleuves et des rivières. Ainsi, le Brésil traverse aujourd'hui une période similaire à celle qu'a connue la France pendant cette période. Au Brésil, nous n'avons pas créé ce signal économique aux consommateurs et nous n'avons pas créé cette quantité de ressources financières capable de rendre possibles tous les investissements nécessaires.

Le deuxième défi c'est la quantité d'eau, en particulier dans le nord-est, mais pas exclusivement. Si l'on compare les précipitations moyennes dans le nord-est brésilien et en France, elles sont similaires, soit environ $600 \mathrm{~mm}$ par an. Alors pourquoi le régime hydrologique est-il différent? Parce qu'en général, dans le nord-est, les sols sont peu profonds, de sorte que la pluie, lorsqu'elle tombe, sature rapidement le sol et l'eau commence à être drainée superficiellement, formant des rivières intermittentes. Et cette eau, si elle n'est pas stockée, s'écoule. Une grande partie du nord-est est donc semi-aride. La seule façon de résoudre ce problème est de mettre en place des travaux hydrauliques et de bonnes pratiques de conservation de l'eau.

Le problème numéro trois concerne les utilisations multiples de l'eau. Il n'y a pas de raison pour que le Brésil n'imite pas ce qui se fait aux États-Unis. Là-bas, la production céréalière du Midwest s'écoule vers le reste du monde au long du Mississippi. Et cela permet de réduire le coût du transport. Au Brésil, nous utilisons l'autoroute, ce qui coûte très cher. Ainsi, l'utilisation des fleuves amazoniens pour transporter la production du centre du Brésil (il s'agit aujourd'hui d'une région de grande production céréalière) et aussi pour produire de l'électricité, sont deux questions mal considérées. Il y a presque une hystérie collective quand on parle de développer des chantiers sur les fleuves amazoniens, nationale et internationale. Cela met beaucoup l'accent sur les conséquences locales de travaux sur un fleuve amazonien, avec des opinions presque toujours mauvaises. On s'intéresse beaucoup à cela et peu aux effets plus globaux. Il faut examiner au cas par cas. Dans certains cas, il me semble logique de ne pas diaboliser les chantiers sur les rivières amazoniennes. Cela est un grand défi pour le développement du centre-ouest et de la région amazonienne.

J.P.C. : Aujourd'hui, la gestion de l'eau au Brésil est extrêmement fragile et l'accès aux services d'assainissement n'est pas encore universalisé. C'est quelque chose d'inacceptable et qui révèle évidemment un décalage entre ce que la loi prévoit et les instruments qu'elle définit dans la réalité, ainsi que le fait que l'assainissement ne soit pas résolu.

Il y a donc une situation intenable. Là où la densité démographique est plus importante, le peu d'eau qui s'y trouve est fortement pollué. Le modèle de 
fonctionnement qui en résulte est celui de captation de l'eau dans des endroits de plus en plus éloignés, indépendamment des coûts et des impacts.

Nous assistons donc à une situation totalement contradictoire, car nous avons un cadre juridique très avancé, mais une réalité très rétrograde. Nous sommes confrontés à une perte croissante de superficie des sources d'eau causée soit par la dégradation du territoire, soit par la contamination qui en résulte.

L.K., G.B. : Y a-t-il un lien entre le changement climatique et la gestion de l'eau ? Quelle est la pertinence de l'impact du changement climatique sur la gestion de l'eau ? Les politiques publiques brésiliennes intègrent-elles dûment la composante climatique?

J.K. : Il existe plusieurs liens entre le changement climatique et l'eau. Tout d'abord, la température augmente, l'évapotranspiration s'accroit, de sorte que les pratiques agricoles exigeront plus d'eau. La consommation d'eau par l'homme aussi, car en général la consommation augmente avec la hausse des températures. Il faut également tenir compte du fait que les régimes hydrologiques changent. La deuxième variable est la pluie. Un changement significatif a été observé dans le cas du Brésil en ce qui concerne les précipitations et le régime des rivières.

Deux exemples intéressant sont le cas du fleuve São Francisco et Paraná. Dans le bassin du São Francisco, 19 des 20 dernières années ont eu un débit moyen inférieur à la moyenne à long terme. D'un point de vue probabiliste, cela semble totalement improbable. Cependant, il se passe quelque chose. Les précipitations ont en effet diminué, de sorte qu'il y a en fait un changement des précipitations dans le bassin du fleuve, mais cela n'explique pas entièrement le changement des débits. Une partie des changements proviennent d'un changement de l'usage des sols. Une partie est donc due au changement des précipitations et une autre au changement d'utilisation $\mathrm{du}$ sol, essentiellement l'augmentation de l'agriculture irriguée. Lorsque vous augmentez l'irrigation, vous augmentez la transpiration, ce qui fait que moins d'eau arrive à la rivière. Dans cet exemple, il y a donc clairement un changement de régime hydrologique qui pourrait être lié au changement climatique. Déjà, dans le sud du Brésil, dans le bassin du fleuve Paraná, il rentre sur le territoire argentin par la centrale hydroélectrique d'Itaipú. Dans le bassin versant d'Itaipú, le débit a augmenté ces dernières années. Et cette augmentation s'explique mal par une modification des précipitations, mais surtout par un changement de l'occupation des sols. Essentiellement la substitution de la forêt native ou du café, par les pâturages.

Lorsque l'on recherche le lien entre l'hydrologie et le changement climatique, il faut veiller à ne pas attribuer au changement climatique certains effets qui, en réalité, trouvent leur origine dans le changement d'utilisation du sol.

Après avoir parlé de température et de précipitation, il faut s'intéresser plus globalement à la modélisation de la circulation atmosphérique qui permet de prévoir le changement climatique. Je pense qu'il y a encore un grand défi scientifique dans cette modélisation. D'un point de vue hydrologique, c'est scientifiquement mal défini.

J.P.C.: Toutes les meilleures études scientifiques révèlent le lien entre les changements climatiques et l'enjeu de l'eau principalement dans la vérification des impacts déjà vérifiés et dans les projections de ce que nous appelons «l'aggravation des événements climatiques extrêmes " qui sont des sécheresses plus sévères et prolongées et des pluies plus intenses et plus concentrées. 
Ces deux éléments, les sécheresses prolongées et les pluies plus intenses, sont des éléments extrêmement compliqués pour la gestion de l'eau. Car, ce qui est attendu et idéal, afin de créer un environnement plus convenable, plus facile à gérer, ce sont des périodes de sécheresse moins intenses, mais bien réparties.

Donc, d'une part, si la gestion des ressources en eau devient de plus en plus complexe, les réservoirs sont menacés de voir leur capacité de stockage réduite. D'autre part, l'excès d'eau a également compliqué les conditions d'entretien des réservoirs. Outre tout le problème de l'envasement causé par les fortes pluies suite à la déforestation qui a eu lieu dans la région des sources. Il s'agit donc d'un facteur aggravant.

Une situation qui est déjà dramatique au Brésil aujourd'hui est la bonne gestion des ressources hydriques. Il n'y a pas de bonne gestion dans un environnement dit «normal » du point de vue climatique, et elle deviendra encore plus difficile dans un contexte d'aggravation des changements climatiques. Toutes les mesures d'atténuation et d'adaptation sont extrêmement en retard si on les compare aux pays qui sont déjà en train de replanifier leur système d'approvisionnement, pour les 20 ou 30 prochaines années. La réalité est que nous ne sommes pas préparés.

L.K., G.B. : Il y a quelques années, de grands centres urbains comme São Paulo et Brasília ont connu d'importantes crises d'eau. Quelles ont été les principales causes de ces crises? Quelles ont été les leçons à retenir?

J.K. : Je parlerai plus spécifiquement du cas de São Paulo, car j'ai vécu au plus près de cette situation. En fait, j'ai assumé mon poste à la Sabesp quand le stock d'eau dans les réservoirs était suffisant pour environ 1 mois, il y avait environ $3 \%$ d'eau réservée. J'ai été invité à préparer l'entreprise à une calamité, qui serait l'effondrement total de l'approvisionnement pour 20 millions d'habitants. Se préparer à cette catastrophe signifiait faire des travaux pour garantir l'approvisionnement d'eau sans interruption des grands hôpitaux, des grands centres pénitenciers et des grands centres d'hémodialyse.

Quelle était la cause de cette situation? Une sécheresse exceptionnelle. Lors de la conception des systèmes d'approvisionnement en eau, les hydrologues utilisent les séries historiques de débits. Vous utilisez la série historique des flux lorsqu'elle est suffisamment longue et São Paulo avait un système conçu pour faire face à la pire sécheresse observée au cours des 90 dernières années. Donc, ce qui est arrivé en 2014-2015 a été une sécheresse extraordinairement rare, laissant le réservoir avec la moitié du taux correspondant à la pire sécheresse qui n'avait jamais eu lieu au cours des 90 dernières années, et $1 / 4 \mathrm{du}$ débit annuel moyen d'affluence. Peu de systèmes d'approvisionnement en eau au monde seraient préparés à cette éventualité. Et São Paulo ne l'était pas, la Sabesp ne l'était pas non plus.

Ce qu'il a fallu faire, c'est une série de travaux extraordinaires et d'urgence pour relier les systèmes de production d'eau, tant sous la forme d'eau brute, tant dans le réseau d'eau potable interconnectant les réseaux de distribution. Un système de contrôle de la pression a été mis en place. La consommation de la population a diminué volontairement et aussi grâce à des incitations économiques, avec une remise pour ceux qui économisaient et une pénalité pour ceux qui consommaient plus. 
La combinaison de ces trois mesures, à savoir les travaux d'interconnexion, le contrôle de la pression sur le réseau et les incitations économiques pour les utilisateurs, a permis de surmonter la crise sans que la catastrophe tant redoutée se produise.

Après cet événement, quelle a été l'attitude de la Sabesp en matière de planification? Si cela s'est produit une fois [la sécheresse], cela peut se reproduire. Il faut tout au moins se préparer à la répétition de ce phénomène, qui semblait rare, mais que nous allons maintenant considérer comme possible. Pour ce faire, de grands travaux d'infrastructure ont dû être faits. Et comme le problème de la qualité de l'eau est lié au problème de la quantité d'eau, les sources existantes dans la RMSP étaient déjà épuisées, soit parce que là où elle peut être utilisée, elle l'est déjà et que là où elle peut être utilisée, la qualité de l'eau est si mauvaise, autour de la collecte et du traitement des eaux usées, qu'elle ne peut être utilisée. La solution a donc été d'apporter l'eau de loin, à $80 \mathrm{~km}$ de la RMSP, dans des sources d'eau de très bonne qualité. Aujourd'hui, São Paulo dispose de ce renfort et je pense que l'approvisionnement est assuré.

J.P.C. : La crise d'eau à São Paulo survenue de 2014 à 2016 est bien expliquée par la météorologie. Il y a eu une relation directe entre la formation d'une barrière qui a empêché l'arrivée des pluies, due au phénomène des rivières volantes. Les pluies qui viennent de la région amazonienne, se heurtent dans les Andes et se dirigent vers la région sud / sud-est du Brésil, l'Argentine, l'Uruguay et le Paraguay. Une barrière s'est formée, ce qui a empêché l'arrivée de ces nuages chargés d'eau et cela a provoqué une interruption des pluies dans cette région, aggravant le problème de la sécheresse dans toute la région sud-est, mais surtout à São Paulo où la crise a été très intense.

En ce qui concerne les leçons, il est important de souligner la fragilité d'une mégalopole comme São Paulo face à des phénomènes météorologiques qui ne sont pas sous notre contrôle.

Le premier facteur clé est la question de l'utilisation de l'eau. La population de São Paulo est-elle préparée à ce genre de situation ? Est-elle informée de cette situation ? Je crois qu'elle ne l'est pas. Il n'y a pas un système d'information permettant à la population d'avoir une perspective et d'être informée. Au contraire, la population, le citoyen, est traité uniquement comme un consommateur. Si vous n'avez pas une population impliquée, si la population n'est pas mobilisée pour chercher des alternatives et collaborer, pour une action momentanée de réduction de la consommation, pour économiser, vous n'avez pas le partenaire principal qui est la société.

Mais nous n'avons pas eu cela pendant la crise, au contraire. La population a été mobilisée. Ce système, qui a débuté en 2014 / 2015 et qui aurait dû être un gain permanent pour la gestion de l'eau, a été abandonné dans un premier temps dès le retour des pluies. Toutes les politiques ont stimulé la réduction et ont fait que les familles investissent dans des systèmes alternatifs d'économie, en utilisant les bonus qu'ils recevaient dans la réduction de la facture. Toutes ces mesures d'encouragement à la consommation consciente ont été annulées sans aucune consultation. C'était donc assez démobilisant. 
Aujourd'hui, l'augmentation de la consommation est encouragée, car toute la génération de ressources pour assurer l'équilibre financier du prestataire de services, en l'occurrence Sabesp, dépend du volume d'eau vendu. Cette leçon n'a donc pas été retenue.

Deuxièmement, il y a la question du besoin de veiller à ce que les sources d'eau, qui sont en fin de compte celles qui fournissent l'eau, soient conservées. Ils sont obligés de prendre de l'eau quelque part. Et, bien sûr, cette crise a montré l'importance des sources d'eau locales, celles qui sont les plus proches des régions de consommation. La crise a démontré le besoin vital que nous avons de disposer de ces sources et comment nous devons les protéger.

Cette crise aurait donc dû inciter les autorités publiques à mettre en place des politiques publiques plus intensives de conservation et de protection des sources d'eau. Ce qui n'est pas arrivé. Au contraire, tous les investissements ont été et continuent d'être faits en matière de garantie de l'approvisionnement en cherchant l'eau dans d'autres bassins, sans tenir compte du coût.

Donc, le citoyen de São Paulo n'a pas intégré de façon permanente une utilisation plus rationnelle et plus économique de l'eau. C'était momentané. Les sources d'eau se dégradent à un rythme accéléré, et cela n'a pas changé. Les leçons n'ont donc pas été tirées.

L. K., G. B. : Quels seraient les principaux goulets d'étranglement pour une utilisation plus durable de l'eau en agriculture?

J.K. : Le principal mécanisme dont dispose le gouvernement pour assurer un usage plus efficace de l'eau est un système de permis d'usage de l'eau, appelé octroi. L'efficacité de ce système suppose un équilibre entre la demande et la disponibilité de l'eau. Par conséquent, cela signifie disposer d'un réseau d'informations hydrologiques qui vous permet d'estimer avec une certaine précision le flux des fleuves, les distributions de probabilité et la quantité d'eau que vous pouvez allouer à un usage productif avec fiabilité. Et l'ANA est l'agence fédérale qui possède les capacités et les connaissances hydrologiques appropriées pour mener à bien une telle tâche. Toutefois, son action se limite aux ressources en eau de la zone de domination de l'Union.

Il n'est pas raisonnable, comme c'est le cas aujourd'hui, que dans certains fleuves d'un même bassin, différentes entités puissent accorder des permis sans faire un bilan global de ce qui est déjà accordé, sans le comparer avec la disponibilité totale du bassin. La mise en place d'un système unifié par bassin fluvial n'est pas encore achevée et si j'étais à l'ANA, ce serait le thème principal auquel je me consacrerais.

J.P.C. : En fait, c'est un mélange de solutions. Le processus d'octroi devrait prendre en considération non seulement le volume d'eau disponible, mais aussi la répartition de ce volume entre les différents acteurs qui l'utilisent. Fondamentalement, la répartition est basée sur le volume d'eau disponible et la demande, et donc une répartition de cette demande en tenant compte des priorités légales. Mais, c'est une erreur. Parce que l'octroi devrait également prendre en compte la question de la garantie de la production d'eau.

En ce sens, on pourrait dire que l'eau est utilisée à sa limite. Cela signifie qu'en étant utilisée à sa limite, elle compromet les conditions environnementales nécessaires - 
non seulement pour l'usage humain sur différents fronts: agriculture, industrie, assainissement public, dévouement des animaux, etc. - L'eau est également nécessaire au maintien des fonctions écologiques, au maintien des écosystèmes qui sont aussi responsables de la qualité même de l'eau. Il s'agit donc d'un cycle fermé.

La question de l'utilisation de l'eau pour l'agriculture est un problème majeur, car elle présuppose et repose sur une intensification de la production agricole. L'intensification de la production agricole est souhaitable, car si vous augmentez la productivité, vous minimisez la nécessité d'étendre les surfaces de terre. Moins il faut élargir la surface, plus il est possible de préserver les milieux naturels, bien que cette équation ne soit pas si simple. Or, le problème est que l'intensification conduit à une intensification et à une expansion intensifiée de l'agriculture. En gros, nous assistons donc à une croissance extrêmement accélérée de l'irrigation. Parce que l'intensification, elle, se développe de plus en plus. Et cette intensification de l'utilisation de l'irrigation compromet la disponibilité de l'eau. Le système le plus rationnel et le plus économique avec une perte d'eau moindre, qui est l'irrigation au goutte à goutte, par exemple, sont des systèmes moins utilisés parce qu'ils sont plus chers et plus complexes.

Et il y a aussi le problème du paiement. Ces subventions et le montant payé pour avoir accès à cette eau sont dérisoires. C'est un coût secondaire, pas un coût majeur. Si je n'ai pas la responsabilité de préserver les sources d'où je tire l'eau, et que je paie également un montant dérisoire pour l'eau que je tire, il est clair que je n'ai aucune incitation à rationner la consommation. Et c'est ce qui se passe dans les grandes exploitations agricoles. L'utilisation de cette eau est gratuite.

Ainsi, l'agriculture, du point de vue de la ressource en eau, devient de plus en plus un facteur de dégradation en raison de l'excès de consommation. Outre la question de la contamination par les pesticides, la question de l'envasement dû à de mauvaises pratiques agricoles de lutte contre l'érosion peut aussi être citée.

L. K., G. B. : Quelle serait la priorité pour promouvoir la sécurité de l'approvisionnement de l'eau au Brésil ?

J. K.: La mise en place, à l'échelle du bassin fluvial, d'un système de permis d'utilisation pour les usagers et de tarification de l'usage des ressources en eau.

J. P. C. : Il s'agit d'un système qui relie un vaste ensemble d'actions et de fronts. Du point de vue de la justice sociale, le numéro 1 serait l'universalisation de l'offre. Mais dans quelles conditions et avec quels risques l'approvisionnement serait-il universalisé ? L'universalisation de l'offre avec des traitements efficaces ? Il n'y a pas de solution à la question de l'eau qui passe par une mesure unique, une mesure miraculeuse. Il est nécessaire de formuler un plan d'action bien structuré qui puisse identifier les différents fronts qui nécessitent des mesures concrètes. Les gens doivent cesser de penser que l'eau vient du robinet et agir de manière plus responsable.

La priorité est la sécurité de l'eau. Nous devons établir la «nouvelle culture de l'eau ", c'est-à-dire une nouvelle culture de la protection de l'eau. L'eau est un bien fini, extrêmement limité, beaucoup plus limité que les gens ne le pensent. Il est nécessaire de changer de conception, de perspective. Cela est notre plus grand combat face au groupe qui a une vision plus technocratique de l'utilisation de l'eau, de la question de la gestion de l'eau. 


\section{BIBLIOGRAPHIE}

Peut-être, si vous insistez sur une mesure unique, serait-ce une obligation pour chacun de changer sa perception de la gestion de l'eau en intégrant la durabilité et l'utilisation de systèmes naturels moins intensifs dans le traitement et la distribution de l'eau.

Agência nacional de águas (ANA). Relatório Conjuntura dos Recursos Hídricos, 2019. http:// conjuntura.ana.gov.br, consulté le 6/03/2020

Civil society working group for the 2030 agenda (CSWG2030). Spotlight Report the 2030 Sustainable Development Agenda: Synthesis III Brazil. https://brasilnaagenda2030.files.wordpress.com/2019/09/ relatorio_luz_ingles_final_v2_download.pdf, consulté le 6/03/2020

Rajendra K., Pachauri, Meyer, Leo, et al., Intergovernmental panel on climate change (IPCC), Climate Change 2014: Synthesis Report, Genève, 2014 https://www.ipcc.ch/site/assets/uploads/ 2018/02/SYR_AR5_FINAL_full.pdf, consulté le 6/03/2020

Intergovernmental Panel On Climate Change (IPCC). Global Warming of $1.5^{\circ} \mathrm{C}$. An IPCC Special Report on the impacts of global warming of $1.5^{\circ} \mathrm{C}$ above pre-industrial levels and related global greenhouse gas emission pathways, in the context of strengthening the global response to the threat of climate change, sustainable development, and efforts to eradicate poverty, 2018. https://www.ipcc.ch/sr15/, consulté le $6 / 03 / 2020$

Marengo, José Antonio. « Água e mudanças climáticas ». Revista de Estudos Avançados, volume 22, $n^{\circ}$ 63, 2008. http://www.periodicos.usp.br/eav/article/view/10294, consulté le 6/03/2020

Un-Water, Water Security \& the Global Water Agenda, 2013. https://www.unwater.org/publications/ water-security-global-water-agenda/, consulté le 6/03.2020

World economic forum (WEF). The Global Risks Report (15 ${ }^{\text {th }}$ Edition), 2020. http://

www3.weforum.org/docs/WEF_Global_Risk_Report_2020.pdf, consulté le 6/03/2020

\section{AUTEURS}

\section{LIVIA KALIL}

Livia Kalil est doctorante contractuelle en Science Politique, IHEAL (CREDA UMR 7227), Sorbonne Nouvelle-Paris 3 en cotutelle au Programme de Sciences Environnementales (PROCAM) de l'Université de Sao Paulo (USP). Coordinatrice du Pôle Brésil de l'Institut des Amériques. liviamaria.kalil@gmail.com

\section{GUILHERME B. CHECCO}

Guilherme B. Checco est doctorant en Changement social et participation politique dans l'Escola de Artes, Ciências e Humanidades de l'Université de São Paulo (EACH/USP) et maître en Sciences environnementales de l'Instituto de Energia e Ambiente (IEE/USP). Chercheur et Coordinateur de projets au Instituto Democracia e Sustentabilidade (IDS). guichecco@hotmail.com 